\title{
Can Common Sense uncover cultural differences in computer applications?
}

\author{
Junia Anacleto ${ }^{1}$, Henry Lieberman ${ }^{2}$, Marie Tsutsumi ${ }^{1}$,Vânia Neris ${ }^{1}$, \\ Aparecido Carvalho ${ }^{1}$, Jose Espinosa ${ }^{2}$, Muriel Godoi ${ }^{1}$ and Silvia Zem- \\ Mascarenhas ${ }^{1}$ \\ 1 Advanced Interaction Laboratory - LIA \\ UFSCar - Rod. Washigton Luis KM 235 - São Carlos - SP - Brazil \\ \{junia, marie_tsutsumi, vania, fabiano, muriel_godoi\}@dc.ufscar.br; \\ silviazem@power.ufscar.br \\ WWW home page: http://lia.dc.ufscar.br \\ 2 MIT Media Laboratory \\ 20 Ames St., 384A Cambridge MA 02139 \\ \{lieber, jhe\}@media.mit.edu \\ WWW home page: http://www.media.mit.edu
}

\begin{abstract}
Cultural differences play a very important role in matching computer interfaces to the expectations of users from different national and cultural backgrounds. But to date, there has been little systematic research as to the extent of such differences, and how to produce software that automatically takes into account these differences. We are studying these issues using a unique resource: Common Sense knowledge bases in different languages. Our research points out that this kind of knowledge can help computer systems to consider cultural differences. We describe our experiences with knowledge bases containing thousands of sentences describing people and everyday activities, collected from volunteer Web contributors in three different cultures: Brazil, Mexico and the USA, and software which automatically searches for cultural differences amongst the three cultures, alerting the user to potential differences.
\end{abstract}

\section{Introduction}

The answer to the title question, according to our preliminary results, is yes. However, to uncover cultural differences is not a simple task. Many researchers have pointed that cultural differences should be considered in the design of interactive systems $[13,7]$. Culture is a shared meaning system which forms a framework for problem solving and behavior in everyday life. Individuals communicate with each other by assigning meaning to messages based on their prior beliefs, attitudes, and values [7]. 
The cultural differences express the "world vision" a group of people have. This vision is expressed in the simple activities that people do everyday. Arguably the most general and widely applicable kind is knowledge about the everyday world that is possessed by most people in a given culture - what is widely called 'common sense knowledge'. While 'common sense' to the ordinary people is related to 'good judgment' as a synonymous, the Artificial Intelligence community uses the term 'common sense' to refer to the millions of basic facts and understandings that most people have. For example, the lemon is sour; to open a door, you must usually first turn the doorknob; if you forget someone's birthday, they may be unhappy with you.

Common sense knowledge, thus defined, spans a huge portion of human experience, encompassing knowledge about the spatial, physical, social, temporal and psychological aspects of typical everyday life. Common sense is acquired from the interaction with the environment. Changing the environment changes the perception of common sense and is one of the reasons why different and diverse cultures exist. This conception of common sense is building ontology about everyday life based on the shared experiences of a community [12].

The challenge is to try to represent cultural knowledge in the machine, and have interfaces that automatically and dynamically adapt to different cultures. While fully implementing this goal is still out of reach, this paper takes some first steps.

We have collected large knowledge bases representing Commonsense knowledge in three cultures: Brazil, Mexico and the USA. Comparison between these knowledge bases gives us a basis for automatically discovering differences between cultures, and finding analogies from one culture to another. Software for cultural comparison is useful in many contexts. For example, by those who want to develop systems focusing on a specific user group (e.g. a teacher who consults the common sense database to prepare a specific instructional content); by those who want to develop systems which use the cultural knowledge stored in the knowledge bases (e.g search engines that consider the context); and by those who want to facilitate communication between people, providing mutual knowledge about their cultures.

In this context, the main purpose of this work, partially supported by TIDIA-Ae FAPESP project, proc no. 03/08276-3, and by CAPES, is to evaluate how the cultural differences can be recognized in the databases that store common sense. For that, we select a theme that frequently appeared in the Brazilian knowledge base food. Considering that eating habits express culture (F1) and common sense affects eating habits (F2), we could say that common sense expresses culture. F1 is taken as true and so we should demonstrate F2.

It is important that the reader understands that we make no claim to have fully described a national culture, or to have fully captured cultural differences. Instead, the goal is simply to get some useful representation of culture and common knowledge, where otherwise the computer would have none. We believe that our contribution is the first attempt to systematically study the extent and nature of cultural differences; to represent cultural differences in machine-readable form; and to present an example of software that searches for such differences and provides inter-cultural translations automatically.

The next section presents how data are collected in the Open Mind Common Sense (OMCS) knowledge bases. To give the reader some idea of how knowledge in the various databases compares, we present some example comparisons for the food 
domain. We then discuss the prototype agent for finding cultural differences, followed by conclusion and future work.

\section{The Open Mind Common Sense Approach for Gathering and Using Common Sense Facts}

Arguably the most general and widely applicable kind of knowledge about the everyday world is the knowledge we typically assume is possessed by ordinary people in a given culture - what is widely called 'common sense knowledge'. One way that $\mathrm{AI}$ has used to represent this knowledge is by simple sentences asserting such facts (pioneered by Doug Lenat's CYC project). For example, a lemon is sour; to open a door, you must usually first turn the doorknob; if you forget someone's birthday, they may be unhappy with you. Common sense knowledge, thus defined, spans a huge portion of human experience, encompassing knowledge about the spatial, physical, social, temporal and psychological aspects of typical everyday life.

Since every ordinary person has the common sense that computers lack, why not involve everyone in building the knowledge base that is necessary to give computers what they need to be able of common sense reasoning? Nowadays, it is easy to reach lots of people through the Internet. For gathering the common sense data some Open Mind Common Sense websites were built. As the name suggests, the Open Mind Common Sense (OMCS) sites are open. Everyone who wishes to help can contribute with his or her knowledge.

The data are stored in the OMCS database as simple statements in natural language. However, for machine use, it is necessary to put them in a representation that allows machines to make practical inferences and analogies. For that, the data are submitted to a natural-language parser that generates a set of normalized nodes that are semantically related, composing a semantic network. A better understanding about how this semantic network is generated is presented by Liu [12].

Once the semantic network is ready, applications can be developed using the common sense knowledge provided by different users.


Figure 1. Common Sense sentences from Portuguese- and English-speaking contributors 


\section{Common Sense and Eating Habits}

In this section, we give the reader a glimpse of what can be compared between the sites. Again, the idea is not to present a definitive scientific survey about such issues as what people actually eat for breakfast in Brazil versus the USA. The knowledge, after all, comes from members of the general public who may have legitimate disagreements or differing experiences about these issues. The idea is that Common Sense collects plausible (rather than completely accurate) answers about these questions, which can lead to plausible assumptions about cultural differences.

Considering the redundancies in our data, we selected categories that appeared in higher frequency in each base. Some of these data were presented in [1]. The categories are presented bellow.

\section{Time for meals}

One of the most common themes in the knowledge bases is time for meals. Table 1 shows what is considered common sense for most of the collaborators.

Table 1.Time for meals.

\begin{tabular}{c|c|c|c}
\hline & Brazil & Mexico & USA \\
\hline Lunch & $11: 30$ to $13: 00$ & $14: 00$ to $16: 00$ & $12: 00$ to $14: 00$ \\
\hline Dinner & $18: 30$ to $20: 00$ & $20: 00$ to $21: 00$ & $18: 00$ to $19: 00$ \\
\hline
\end{tabular}

Here it is interesting to note that meals in Mexico are the latest ones. Although in Brazil and the USA, meals happen at a similar hour, in Mexico it seems to be common to have lunch after 14:00.

What do people eat in each meal?

Differences between what is eaten in each meal also can be noticed. Table 2 shows what seems to be considered common sense about what to eat in each meal.

It is possible to notice that Brazilian people prepare lighter food at breakfast. Also Mexican people seem to like food make with flour. Concerning desserts, Brazilian people associate ice cream as something cooling, and are reluctant to eat it in Brazil's (relatively mild) winter. On the other hand, American people seem to prefer pies and other baked desserts.

Table 2. What do they eat in each meal?

\begin{tabular}{c|c|c|c}
\hline Breakfast & Brazil & Mexico & USA \\
\hline Lunch & $\begin{array}{c}\text { rice, } \\
\text { beans, } \\
\text { meat, } \\
\text { with hot sauce }\end{array}$ & $\begin{array}{c}\text { chicken with } \\
\text { mole, roast } \\
\text { meat, pastries, } \\
\text { shilad, egg } \\
\text { chiles, } \\
\text { barbacoa, tacos }\end{array}$ & $\begin{array}{c}\text { hambargels, } \\
\text { dog, pizza, } \\
\text { sandwiches }\end{array}$ \\
\hline Dinner & $\begin{array}{c}\text { rice and } \\
\text { beans, }\end{array}$ & $\begin{array}{c}\text { tamales and } \\
\text { atole, }\end{array}$ & $\begin{array}{c}\text { steak and eggs, } \\
\text { baked chicken, }\end{array}$ \\
\hline
\end{tabular}




\begin{tabular}{|c|c|c|c|}
\hline & $\begin{array}{c}\text { soup, } \\
\text { salad, } \\
\text { sandwich }\end{array}$ & $\begin{array}{c}\text { quesadillas, } \\
\text { coffee and } \\
\text { cookies, bread } \\
\text { with bean }\end{array}$ & $\begin{array}{c}\text { clam chowder, } \\
\text { mashed } \\
\text { potatoes }\end{array}$ \\
\hline Dessert & $\begin{array}{l}\text { ice cream, } \\
\text { fruit, } \\
\text { candy }\end{array}$ & $\begin{array}{l}\text { rice with milk, } \\
\text { churros with } \\
\text { chocolate, nuts } \\
\text { with honey } \\
\text { (crowbar), } \\
\text { sweet coconut }\end{array}$ & $\begin{array}{c}\text { pumpkin pie, } \\
\text { apple pie, ice } \\
\text { cream, cheese } \\
\text { cake }\end{array}$ \\
\hline
\end{tabular}

\section{Food for special occasions}

Christmas and parties were topics that collaborators remembered too. Table 3 shows the main types of food cited for this occasions. It is interesting to notice that in Brazil and México it seems to be common have salty food for Christmas while in the USA sweet dishes seem to be more appreciated. On the other hand, beer seems to be appreciated at parties in all three countries.

Table 3. Food for special occasions.

\begin{tabular}{c|c|c|c}
\hline Party & Brazil & Mexico & USA \\
\hline Christmas & $\begin{array}{c}\text { snack, candy, } \\
\text { cake, meat, } \\
\text { beer }\end{array}$ & beer, tequila & beer, vodka \\
& $\begin{array}{c}\text { turkey, pork, } \\
\text { lamb }\end{array}$ & $\begin{array}{c}\text { romeritos, } \\
\text { codfish, } \\
\text { spaghetti }\end{array}$ & $\begin{array}{c}\text { cranberry } \\
\text { sauce, } \\
\text { pineapple } \\
\text { salad, frozen } \\
\end{array}$ \\
& & Christmas \\
& & Pudding \\
\hline
\end{tabular}

\section{Using Cultural Knowledge in Interactive Applications}

We believe the cultural differences stored in the common sense bases can be helpful in (a) helping those who want to consider these differences in the development of interactive systems; (b) facilitating the interaction of different users by applications that use this common sense; and (c) facilitating the communication between people. Here we point out how developers involved in the situations cited above can use the cultural differences stored in common sense knowledge bases.

Developing systems considering cultural differences: Human Computer Interaction research raises further questions about how to understand culture and how it can and should affect user-interface design. Attributes as attraction, dynamism, activity, level of expertise, faith, intentions, locality, social validation, preferences, and scarcity have different weightings in different cultures [2]. Consequently, user-interface developers face further challenges [13]. Many questions still persist while talking about considering cultural differences in the design of interactive systems. Marcus [13] raises some questions: Are our notions of 
usability culturally biased? How should culture differences relate to persuasion and establishment of trust in Web sites and Web-based applications? How should culture dimensions relate to established dimensions of intelligence and change your thinking about online help, documentation, and training? How do culture differences relate to new insight about cognition differences? Do these differences change your thinking about user search strategies, mental models, and navigation? The only consensus seems to be that these attributes have different values and are key characteristics of the cultures to which they belong [4]. Despite the importance of these questions, some developers still face an uphill battle to get budgets for culture-oriented research and development accepted, to find and allocate the human resources, and to achieve project success [13]. In this context, collecting these "world views" and making them available for everyone that wants to develop a user-interface, can be expensive and laborious. The use of the Internet and the collaboration of millions of people allow knowledge bases to reflect actual cultural knowledge without cost, as anyone can have access to the database at the sites.

Developing systems which consider cultural differences: As the complexity of computer applications grows, it may be that the only way to make applications more helpful and avoid stupid mistakes and annoying interruptions is to make use of common sense knowledge. Cellular telephones should know enough to witch to vibrate mode if you're at the symphony. Calendars should warn you if you try to schedule a meeting at $2 \mathrm{AM}$ or plan to take a vegetarian to a steak house. Cameras should realize that if you took a group of pictures within a span of two hours, at round the same location, they are probably of the same event [8]. In the web context, the necessity of using common sense knowledge becomes even more evident. The number of web pages available on Internet increases day after day, and consequently, finding relevant information becomes more and more a difficult task [3]. Also, Web Search tools do not do a very good job of discerning individuals' search goals [16]. However, when we consider communities of people with common interests, it is possible to improve the quality of the query results using knowledge extracted from common sense databases and observing behaviors of people of same culture. When a user submits a query, the cultural aspects suggest specific information exploiting previous observations about the behavior of other users when they asked similar queries. Different users may merit different answers to the same query [3]. As cultural differences can be detected in common sense bases, search engines that attempt to leverage common sense have a great opportunity to reflect cultural differences in their results.

Developing systems which facilitate communication between people by showing cultural differences: Communication between people from different cultures is a field which presents many interesting aspects. To show that common sense can help showing the cultural differences, a prototype of a mail client was developed. The application has an agent that keeps watching what the user is typing, while makes commentaries on the differences in the grounding that can lead to possible misunderstandings. The system also uses these differences to calculate analogies for concepts that evoke the same social meaning in those cultures. We focus this prototype on the social interaction among people in the context of eating habits, but it could scale to other domains. The system's interface has three sections, as can be seen in Figure 2. The first one - at the upper left - is the information for 
the email addresses and the subject, the second one - at the upper right - is where the agent posts its commentaries about the cultural differences and the third part - the lower part - is the body of the message. The second section has four subsections: the upper one shows the analogies that the agent found and the other three show the data that are not suitable for analogy. For example, in our screen shot, the third label for the Mexican culture - Mexicans thinks that dinner is coffee and cookies - and the second for American culture - Americans think that dinner is baked chicken - cannot make a meaningful analogy even if they differ only in one term.



Figure 2. A screen shot of the system.

In order to make the cultural analogies, the system uses four semantic networks. The OMCSNet [10] semantic network (OMCSNet.OM), which was mined from the Open Mind corpus, is used as the core engine because it provides tools for context expansion and is especially designed for working with Open Mind Common Sense databases. The other three databases are culturally specific; they have knowledge about the Brazilian, Mexican and North-American culture - these semantic networks are called OMCSNet.BR, OMCSNet.MX and OMCSNet.US respectively. The OMCSNet.BR was built from data mined from the Brazilian Open Mind Common Sense database. In the American and Mexican cases, the statements were already in English language. In the Brazilian case, the statements were originally in Portuguese. For this project, a small group of statements related to eating habits were selected and freely translated to English to be parsed.

The calculation of cultural analogies is divided into eight steps.

Data retrieval (step 1): The first thing that the system does is to use the NLP package MontyLingua [9] to get the relevant concepts of the mail. This information is presented as tuples of (verb subject direct_object indirect_object). In the example above, the NLP tool produces the output ("have" "I" "dinner" "my place").

Context retrieval (step 2): Then, we use each direct and indirect object of the tuples from the previous step to query the OMCSNet.OM for the relevant concepts. Querying this network first gives us some query expansion of "culturally 
independent" relations. That is, the base OMCSNet.OM, as our largest and most diverse collection, is taken as the "standard" to which the other databases are compared. Since OMCSNet.OM itself has some cultural bias, it would be better, once we have collected enough knowledge bases in other languages, to create a "worldwide" knowledge base by removing all culturally-specific statements, as a basis for comparison. The result of this query is used to get the context in the culturally specific nets. At the end of this stage the output was ranked using two criteria: the first one prefers the concepts resulting for the cultural databases, and the second is to rank first the concepts that come from the part of the email that the user has just written. This helps to address the relevance effectively by giving preference to the important topics of each culture, and in the recent topics of the mail. This process brings concepts as lunch, food, meal, and salad, that are in the semantic neighborhood of dinner.

Node retrieval (step 3): In this step, we get the tuples whose nodes are in the context of the mail from OMCSNet.MX and OMCSNet.US. The output of this step is the pieces of common sense knowledge for the Mexican and American culture (see Figure 3). At this point we have everything the databases have about the eating habits in both cultures. For example: the output from OMCSNet.MX has the following, among others: ['TakeTime', 'dinner', 'between 8:00 PM and 9:00 PM'], ['KindOf', 'dinner', 'light meal'], ['IsA', 'dessert', 'rice with milk'], ['IsA' 'food' 'chocolate']; and from OMCSNet.US has: ['TakeTime', 'dinner', 'between 6:00 PM and 7:00 PM'], ['KindOf', 'dinner', 'heavy meal'], ['IsA', 'dessert', 'pumpkin pie'], ['IsA' 'food' 'chocolate'].

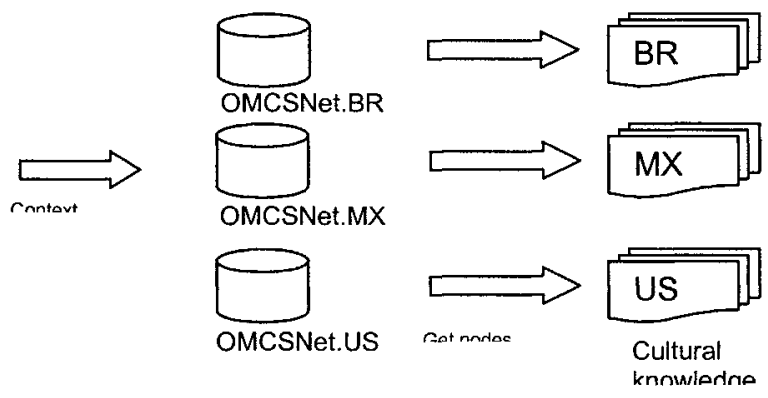

Figure 3. The node retrieval operation.

Relevance of the nodes (step 4): By comparing each node with the cultural sets of knowledge in the previous step we can get its relevance. For this operation, the SIM operation from Cohen's WHIRL [12] is used, as in Figure 4. The interesting part about WHIRL is that it is a system that effectively interleaves inference with retrieval. This operation allows getting the similarity for each node in one set with all the elements of the other set and always maps to a number between zero and one.

Calculation of analogies (step 5): If the value of one node and the semantic relations in the tuples of one set are equal to the tuples of the other cultural set, then the unmatching concept is an analogy between the two cultures that are being considered. In addition, the semantic relation is analyzed in order to avoid irrelevant analogies. These analogies are ranked using the similarity between the two nodes. If 
two different pairs of tuples allow the same analogy, the values of the relevance scores are added. In our example set, the only nodes that are suitable for analogy are the nodes that talk about the 'KindOf' meal the dinner is in both cultures. This process is similar to Dedre Gentner's classic Structure Mapping analogy method.

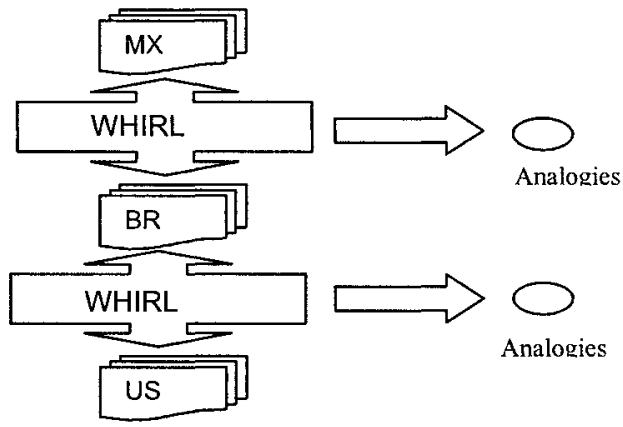

Figure 4. Calculation of the relevance and the analogies.

Calculate the nodes to display (step 6): The nodes are sorted using the relevance of their context and their similarities produced by the SIM operator.

Chose the information to display (step 7): The nodes ranked higher are chosen to be displayed. First, we choose the nodes to calculate analogies and then the rest of the nodes, the former nodes give information about things that are different, but do not have a counterpart in the other culture, or are grounding information that makes no sense for an analogy [6].

Map the concept to English (step 8): For each semantic relation in the net, a custom template that maps its information to English sentences was created and applied before displaying the information. For the analogies, an additional template is used to explain why the system made this analogy. In Figure 2 we show only the top four concepts that are displayed after applying the template.

\section{Conclusions and Future Works}

This work has presented some first steps in the modeling and use of cultural differences in interactive applications. This way, we answer affirmatively to the question proposed in the title of this paper. We model cultural differences by comparing knowledge bases of Commonsense statements collected from volunteer Web contributors in various cultures. We explore applications by those who want to focus on a specific user group; by those who want to develop systems which use the cultural knowledge stored in the knowledge bases; and by those who want to facilitate communication between people, providing mutual knowledge about their cultures. This work is part of a larger effort to model Commonsense knowledge. In [8], we present many applications that have built using the OMCS, ConceptNet, and related tools, for providing intelligent defaults in interactive systems and mapping from user goals to actions. Future work will also include the investigation of cultural expressions in Open Mind Common Sense considering a larger number of facts. Also other domains will be studied in order to verify the cultural differences besides eating habits domain. While we have not yet conducted formal user tests with the 
email application, informal feedback from users shows that, while the absolute accuracy of suggestions is not high, users do appreciate the occasional useful suggestion and it is not excessively distracting even when suggestions are not relevant. We hope developers of interactive systems use the knowledge about culture stored in Open Mind Common Sense databases in order to facilitate the interaction between humans and computers.

\section{References}

1. Anacleto, J.; Lieberman, H; Tsutsumi, M.; Neris, V.; Carvalho, A.; Espinosa, J.; ZemMascarenhas, S. Using Common Sense to Recognize Cultural Differences. Submitted as a work-in-progress paper to $\mathrm{CHI} 2006$.

2. Bailey, B.P., Gurak, L.J., and Konstan, J.A. An examination of trust production in computer-mediated exchange. Proc. 7th Human Factors and the Web 2001 Conference www.optavia.com/hfweb/7thconferenceproceedings.zip/bailey.pdf. Last visited in Jan, 06.

3. Birukov, A., Blanzieri, E, Giorgini, P. Implicit: An AgentBased Recommendation System for Web Search. Proc. AAMAS'05 (July 25-29,2005, Utrecht, Netherlands).

4. Choi, B., Lee, I., Kim, J., Jeon, Y. A Qualitative Cross-National Study of Cultural Influences on Mobile Data Service Design. Proc. CHI 2005.

5. Cohen, William W. WHIRL: A word-based information representation language. Artificial Intelligence (2000) 163-196.

6. Falkenhainer, B., Gentner, D. The Structure-Mapping Engine. Proceedings of the Fifth National Conference on Artificial Intelligence. (1986)

7. Khaslavsky, J. Integrating Culture into Interface Design. Proc CHI 1998 p 365-366.

8. Lieberman, L., Liu, H., Singh, P., Barry, B. Beating Common Sense into Interactive Applications. American Association for Artificial Intelligence, Winter 2005, p 63-76

9. Liu, Hugo. MontyLingua: An End-to-End Natural Language Processor for English. (2003)

10. Liu, Hugo and Singh, Push. OMCSNet: A Commonsense Inference Toolkit. MIT Media Lab Society Of Mind Group Technical Report SOM02-01 (2002) 272-277.

11. Liu, H.; Singh, P. Commonsense Reasoning in and over Natural Language. Proc. 8th KES 04. http://web.media.mit.edu/ push/CommonsenselnOverNL.pdf. Last visited in Jan, 06.

12. Liu, H; Singh P. ConceptNet: A Practical Commonsense Reasoning Toolkit. BT Technology Journal, v. 22, n. 4, p. 211-226, 2004. http://web.media.mit.edu/ push/ConceptNet-BTTJ.pdf. Last visited in Jan, 06.

13. Marcus, A. Culture Class vs. Culture Clash. Proc. Interactions 2002 (June, 2002), 25 p.

14. Singh, P. The OpenMind Commonsense Project. KurzweilAI.net, 2002. Available in: :< http://web.media.mit.edu/ push/OMCSProject.pdf $>$. Last visited in Jan, 06.

15. Spink, A., Ozmutlu S., Ozmutlu H.,Jansen B. J. U.S. VERSUS EUROPEAN WEB SEARCHING TRENDS. SIGIR Forum. Fall 2002, Vol. 36, No. 2, p 32-38

16. Teevan, J., Dumais, S. T., Horvitz, E. Personalizing Search via Automated Analysis of Interests and Activities. Proc. SIGIR '05 (August 15-19, 2005, Salvador, Brazil). 